PNL-SA-21238

"EFFECTS OF SURFACE DEFECTS ON THE LOCAL ELECTRIC FIELD IN INHOMOGENEOUS MEDIA"

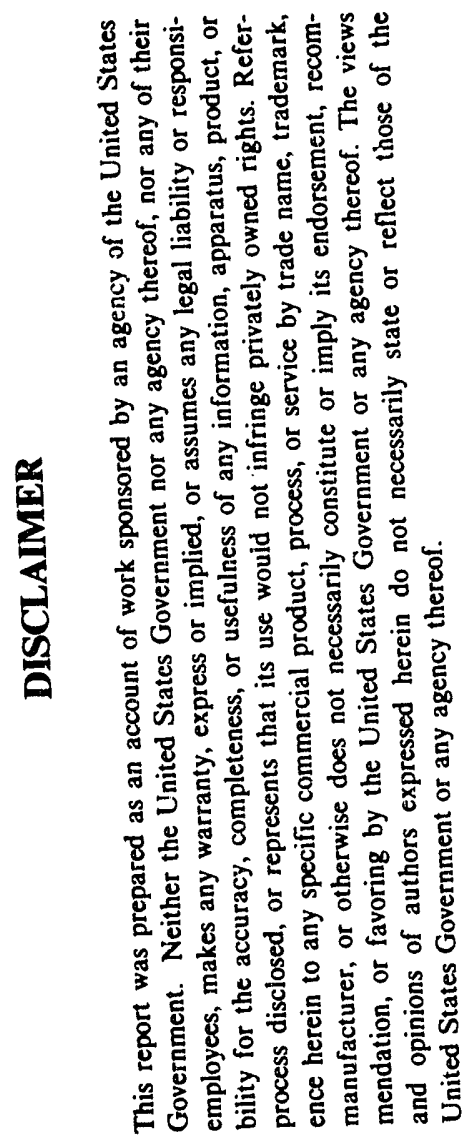

K. F. Ferris

S. M. Risser

February 1993

Presented at the
RECr.'.T

APR 1 i 1993

$05 \% 1$

1992 Fall Meeting

November 30 - December 4, 1992

Boston, Massachusetts

Prepared for

the U.S. Department of Energy

under Contract DE-ACO6-76RLO 1830

Pacific Northwest Laboratory

Richland, Washington 99352

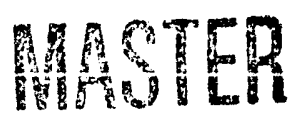




\title{
EFFECTS OF SURFACE DEFECTS ON THE LOCAL ELECTRIC FIELD IN INHOMOGENEOUS MEDIA
}

\author{
KIM F. FERRIS* AND STEVEN M. RISSER** \\ * Materials Science Department, Pacific Northwest Laboratory, Richland, WA 99352 \\ **Department of Chemistry, University of Pittsburgh, Pittsburgh, PA 15260.
}

\begin{abstract}
Surface defects both focus the local electric field and enhance its intensity in the vicinity ' the defect. Using a finite element model of an inhomogeneous dielectric film, we have examined the relationship between the defect shape and local electric field in the film and defect. We find that raised defect regions that are peaked have the largest local fields while the slope of removed regions has little effect on the field in raised defects. Both peaked and flat surface defects can lead to large enhancements of the local electric field above that predicted by effective medium approximation (EMA) methods.
\end{abstract}

\section{INTRODUCTION}

While many properties of dielectric films can be adequately described by volume fractions of the film components, others are strongly influenced by the inhomogeneity produced by the film microstructure. Microstructural details, such as the columnar growth seen from CVD processes [1] and the spherical grains in sol-gel generated coatings [2], have been shown to affect the accuracy to which we can determine the dielectric properties of films.

Previously, the most common methods used to model the dielectric properties of inhomogeneous media, have been based upon scaled macroscopic properties of the medium. The interaction of those characteristics generated by the microstructure are approximated by a surrounding 'effective' medium in these methods. Thus, these theories can provide little information about the microscopic properties of the medium and are not particularly relevant for examining properties relating to the microstructure.

In principle, the local electric field in an inhomogeneous medium can be directly determined from Maxwell's equations [3], however, the complex boundary conditions imposed by the microstructure prevent analytic solution for the field. To address this question, we developed a method to numerically calculate the static local electric field in an inhomogeneous medium. This method uses the self-consistent solvtion for both the local field and polarization within a lattice of cubic volume elements, and has been discussed in 
previous work $[4,5]$. In this work, we use this model to simulate defects on the surface of a homogeneous dielectric film. We show the effect of defect shape on the local electric field within the film and defect, providing information on the types of defects leading to high electric field intensities.

\section{RESULTS AND DISCUSSION}

The large spatial variation of the dielectric function in a film caused by volume defects has been shown to lead to large fluctuations in the local field intensity $[4,6]$. The presence of defects at the surface of the film can also produce large variations in the local field from the field that would exist without the defect, with the variations of the field directly related to the shape of the defect.

The location of these large variations in the local electric field can be readily seen in a cross section of the lattice structure showing the local fields. It is important to note that these details of microstructural information would not be available from effective medium approximation (EMA) methods. In Figure 1, we show the enhancements to the local fields for a cross section through the center of the lattice containing four different shapes of surface defects. The illustrated fields are the calculated field for the inhomogeneous film minus the field for that type of lattice element in a perfect lattice. The lattice for these calculations consisted of 12 layers of $31 \times 31$ cubes, with 5 layers of vacuum above the lattice, although we show only the region near the defect. The dielectric constant of the medium (shaded region) was taken to be 2.0. The four defect shapes in Figure 1 differ by whether the raised region of the defect is peaked ( $1 \mathrm{a}$ and 1c) or flat (1b and 1d), and by whether the removed defect region is sharp (1a and $1 b)$ or sloped (1c and 1d).

In general, sloping regions whether they be a peaked defect or sloping vacant site, cause large enhancements in the local field intensity. The peaked defects (Figs. 1a and 1c) exhibit larger local fields both in the peak and in the volume directly below it. These fields are the largest observed in any of the lattice elements. This effect is independent of whether the removed region is sharp or sloped. When the removed region is sloped, the field in the defect is larger, although this enhancement is minimal in the raised region of the defect. The local field in the removed region (vacuum) has the lirgest deviation from the normal vacuum field when the removed section has a sharp siope.

Again, we note that including of the shape of the defect structure in our model and allowing the explicit interaction of these eiements can cause large variations in the local field. For the specific cases illustrated here, the presence of isolated surface defects would not perceptibly change the void fraction of the film. In turn, EMA methods would most 
probably predict an electric field value close to the idealized lattice background. Thus, the large enhancements in the defect regions are specific deviations of the microscopic information from the EMA approach. Where concentrations of electric field would be a critical variable; these regions of surface 'roughness' would be good candidates for initial study.

\section{CONCLUSIONS}

We have used a finite element method to directly evaluate the local field within inhomogeneous media, and have determined the change in the local electric field resulting from the presence of surface defects of various shapes. We find that regions raised above the film surface have larger local fields, with the field largest when the raised region is peaked. The presence of a removed region has little effect on the local field in the surrounding film, but does enhance the field in the neighboring raised region.

\section{Acknowledgments}

The Pacific Northwest Laboratory is operated by Battelle Memorial Institute for the U.S. Department of Energy, under contract DE-AC06-76RLO 1830. SMR acknowledges support by the National Science Foundation (Grant \# CHE-9106689) and the Defense Advanced Research Projects Agency (Grant \# 91-NC-146) which is administered by the Air Force Office of Scientific Research through agreements with the National Aeronautics and Space Administration.

\section{REFERENCES}

[1] R.E. Klinger and P. Swab, SPIE Proceedings, R.I. Seddon, Ed., 678, 41 (1986).

[2] W.S. Frydrych, G.J. Exarhos, K.F. Ferris, N.J. Hess, Mat. Res. Soc. Symp. 121, 343 (1988).

[3] W. Jackson, Classical Electrodynamics (J. Wiley and Sons, New Y'ork, 1962).

[4] S.M. Risser and K.F. Ferris, Mat. Lett. 14, 99 (1992).

[5] S.M. Risser and K.F. Ferris, Mat. Res. Soc. Symp. 193, 687 (1990).

[6] S.M. Risser and K.F. Ferris, Laser Induced Damage in Optical Materials: SPIE Proc. 1441, 262 (1991). 


\section{FIGURE CAPTIONS}

Figure 1 - Enhancements of the Local Electric Fields for Surface Defects (a) Peak-Sharp, (b) Flat-Sharp, (c) Peak-Slope, and (d) Flat-Slope. 

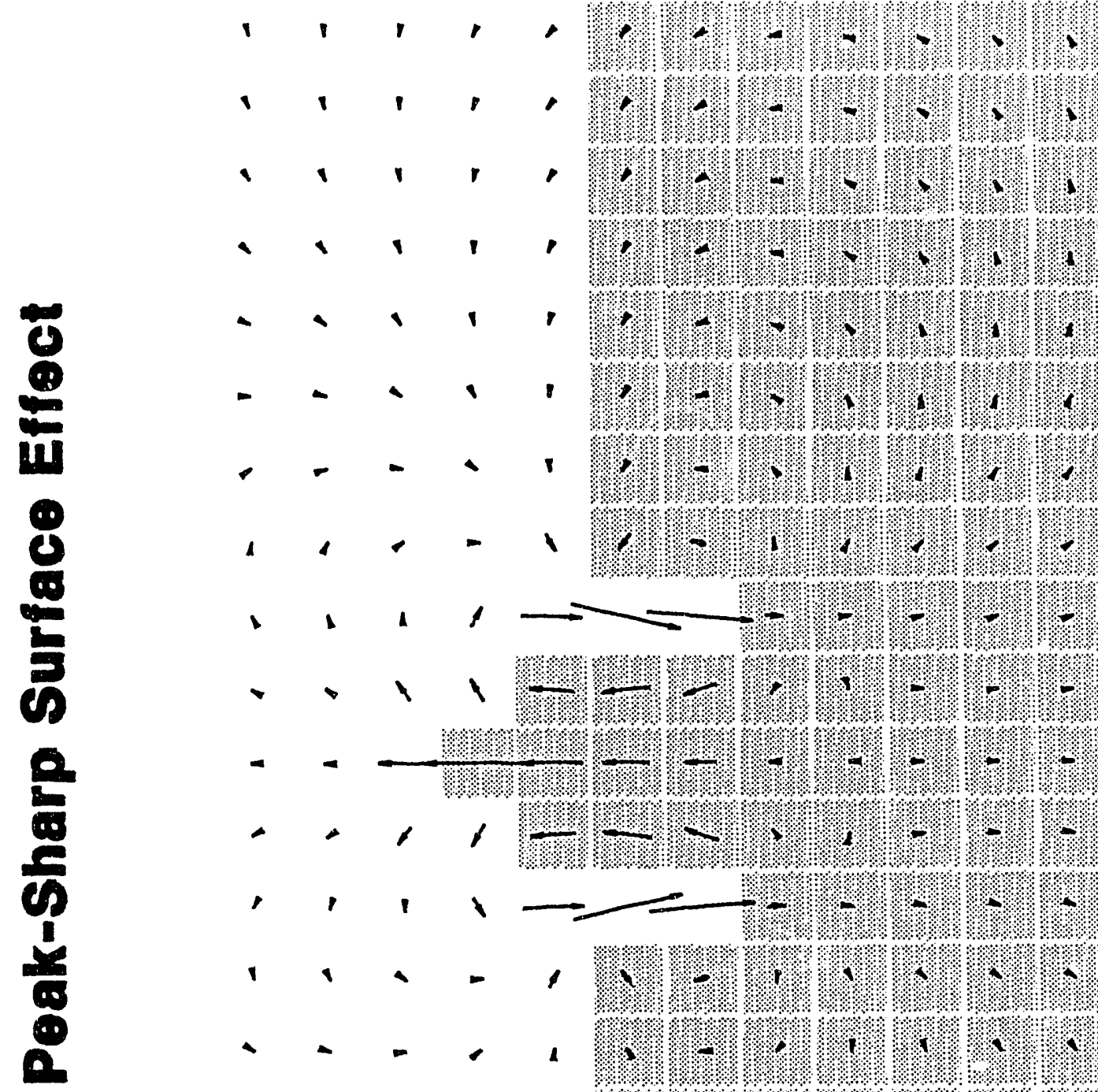

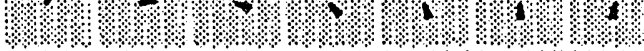
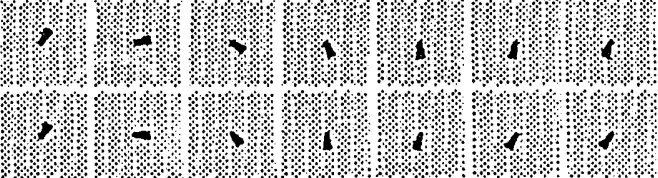

d $, 1,+$,
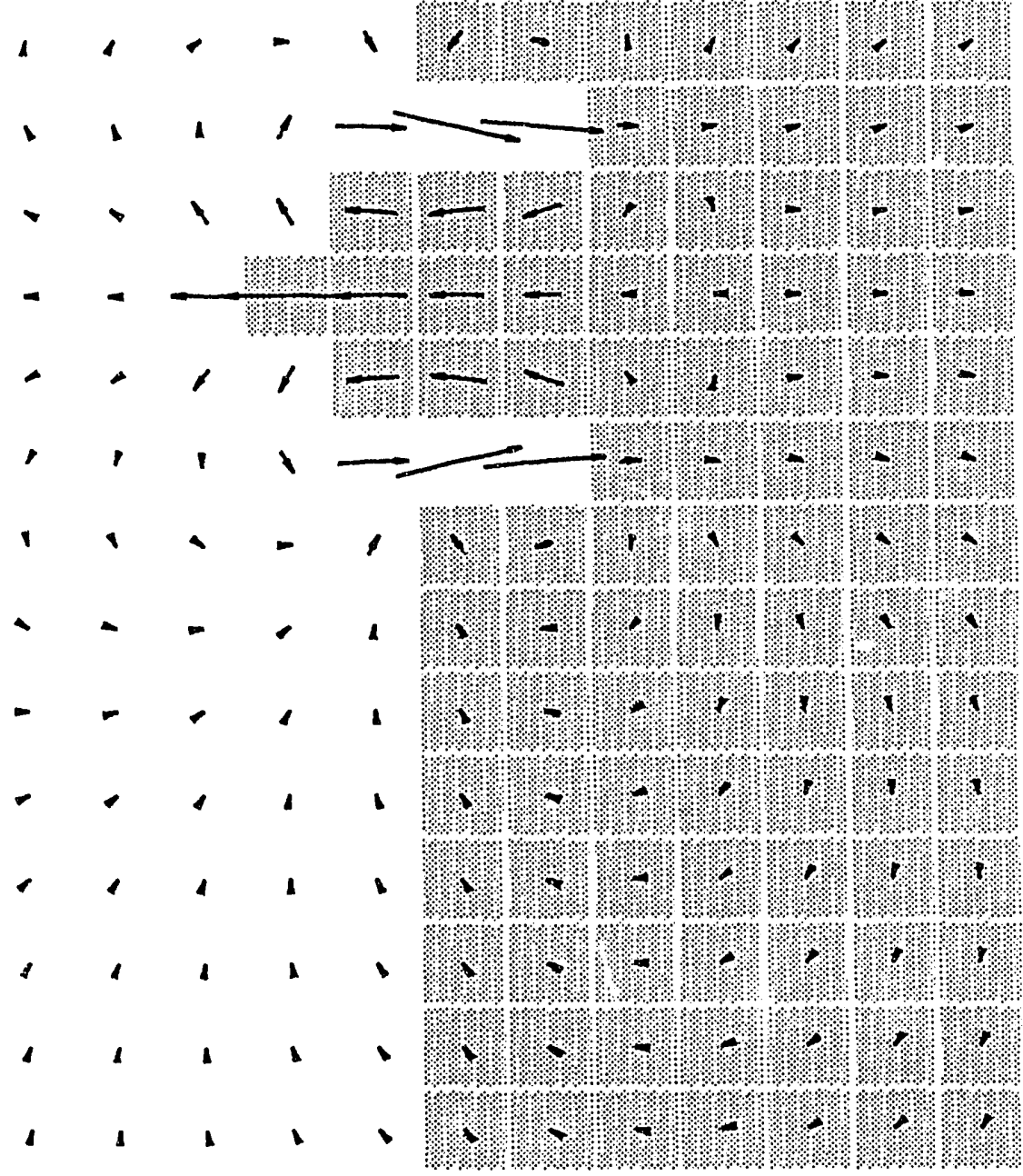


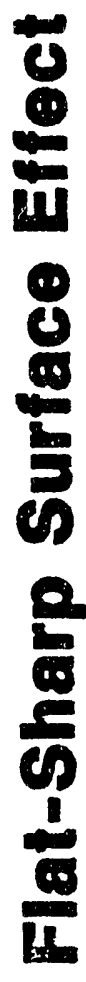
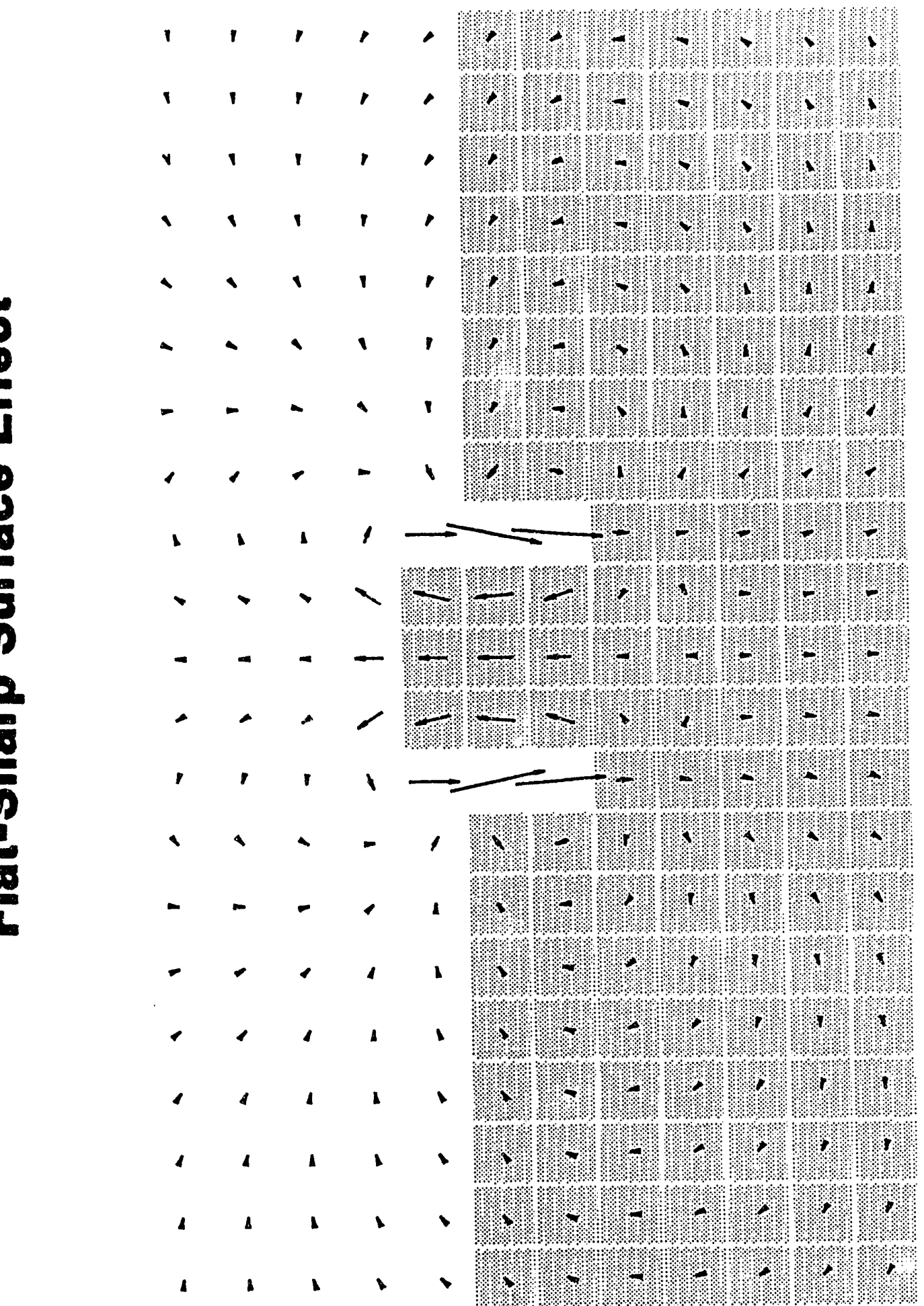
0
0
0
0
0
0
0
0
0
0
$\frac{1}{0}$
0
0

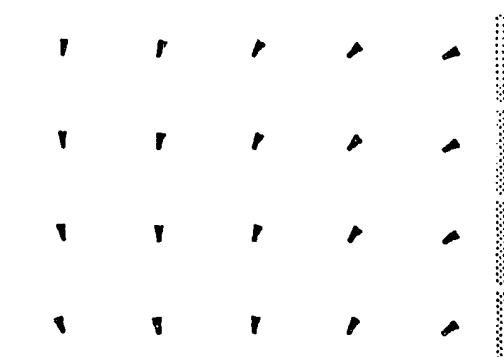

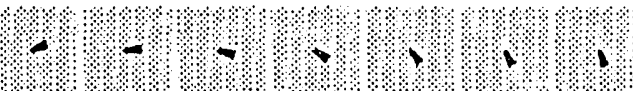

?

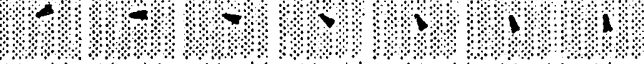

tof

t

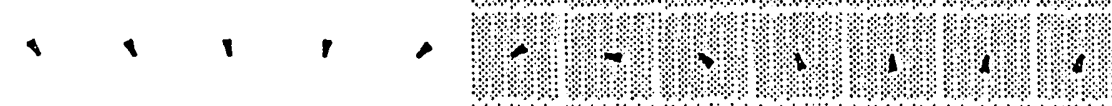

- I $\quad$.

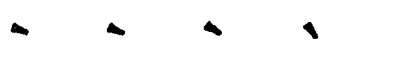

t.

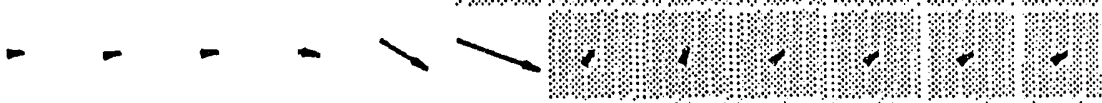

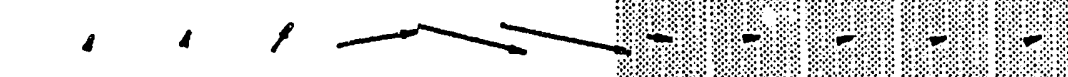

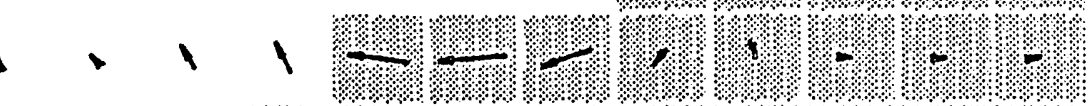

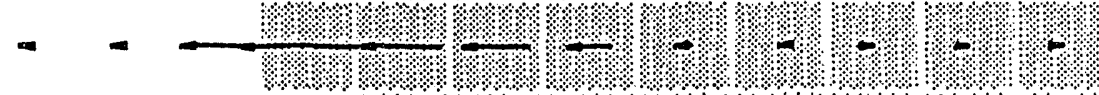

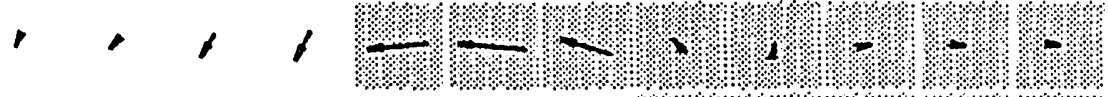

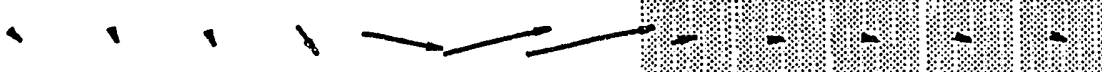

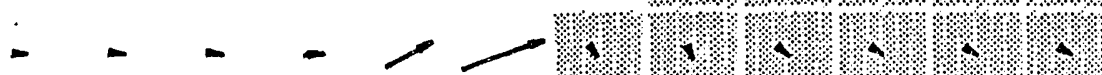
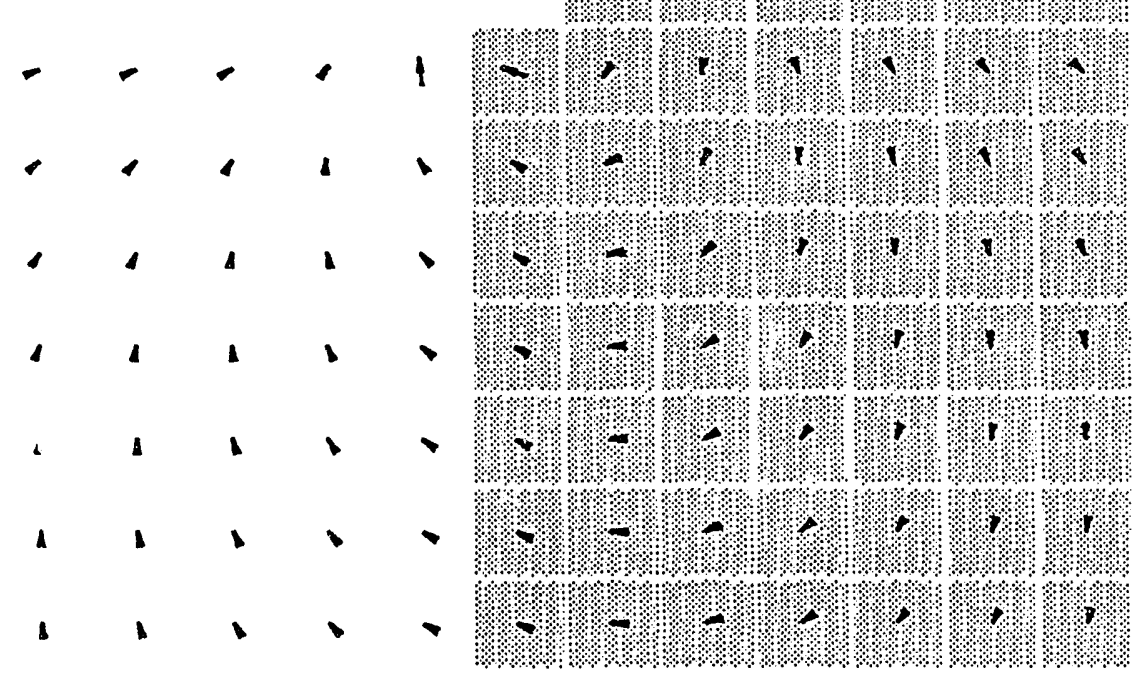


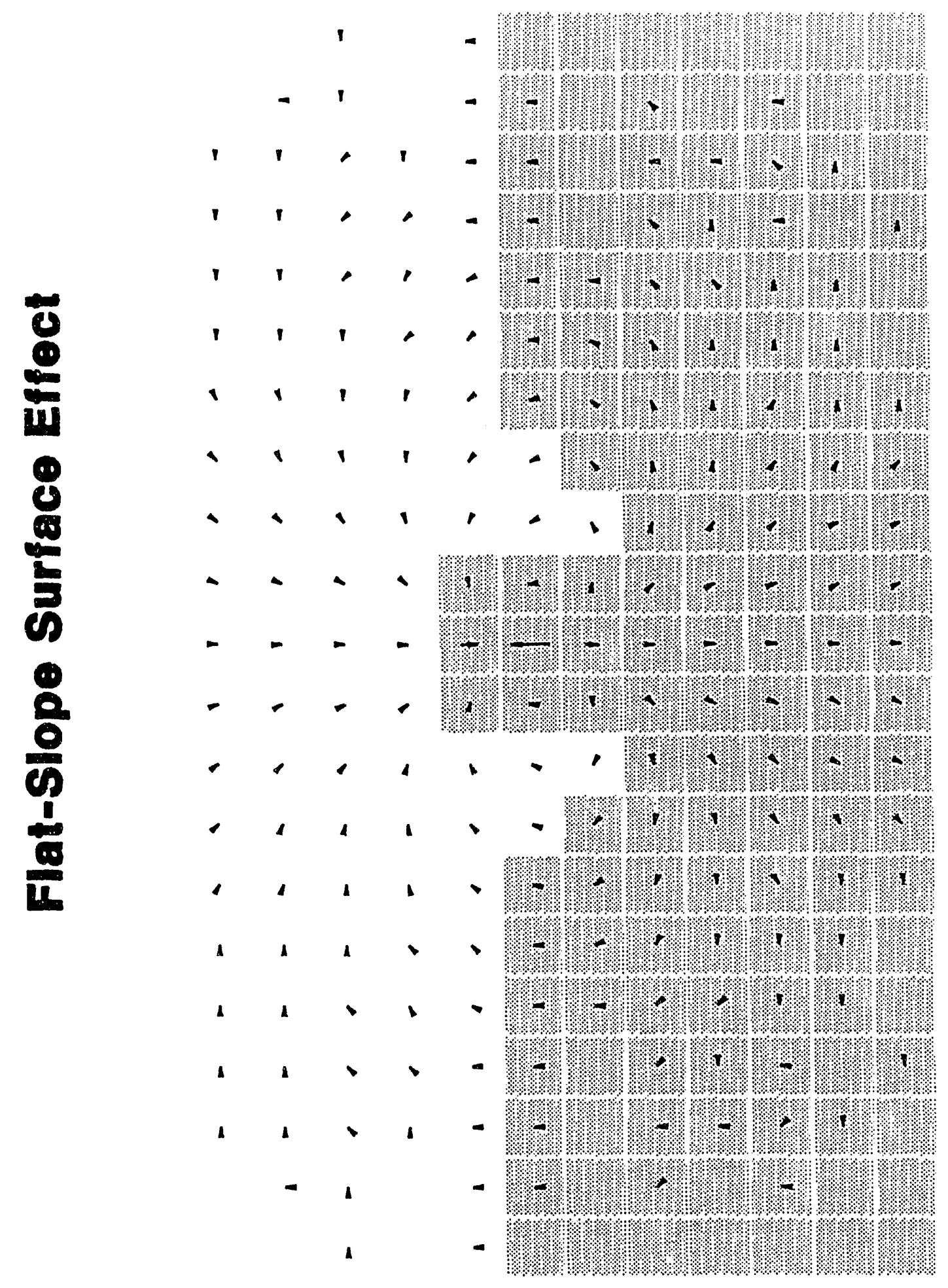



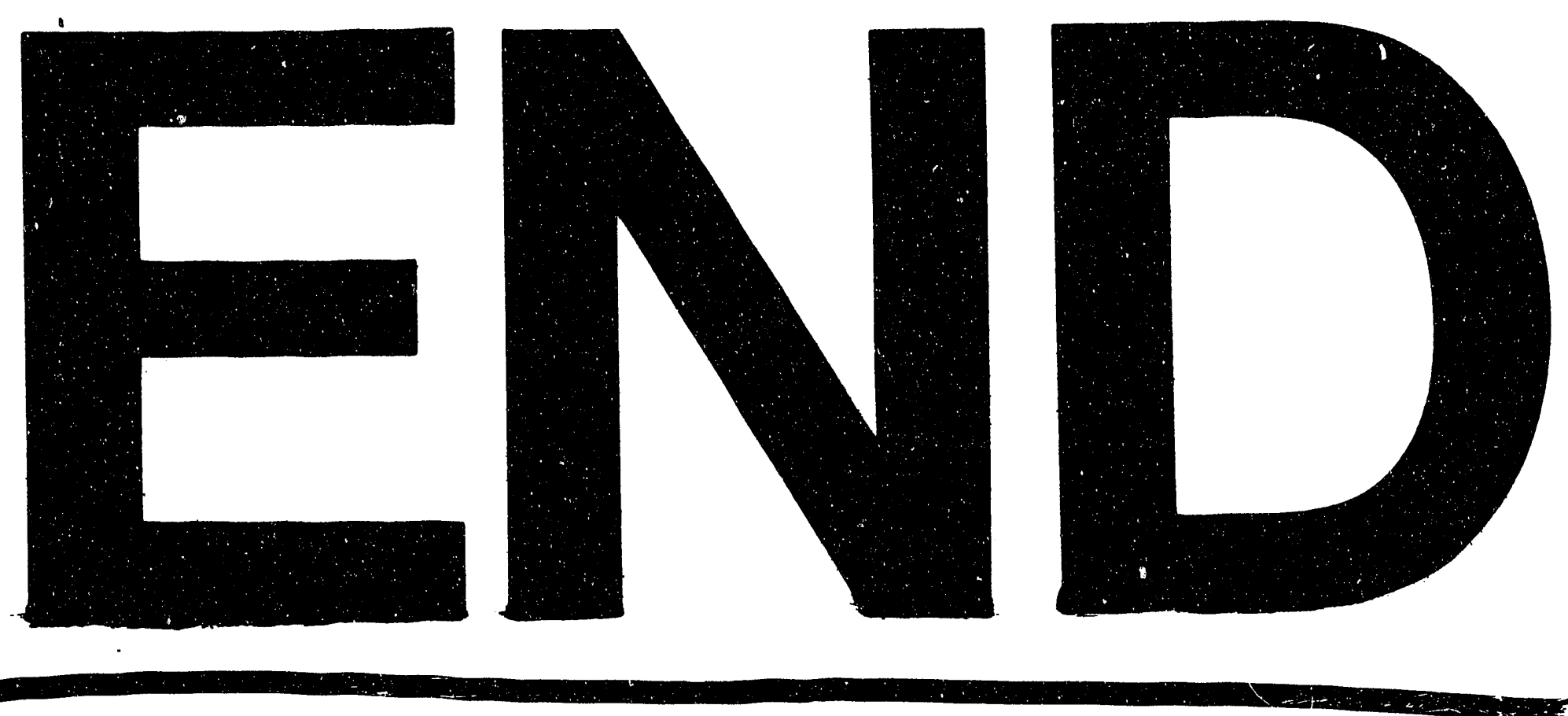

$x$
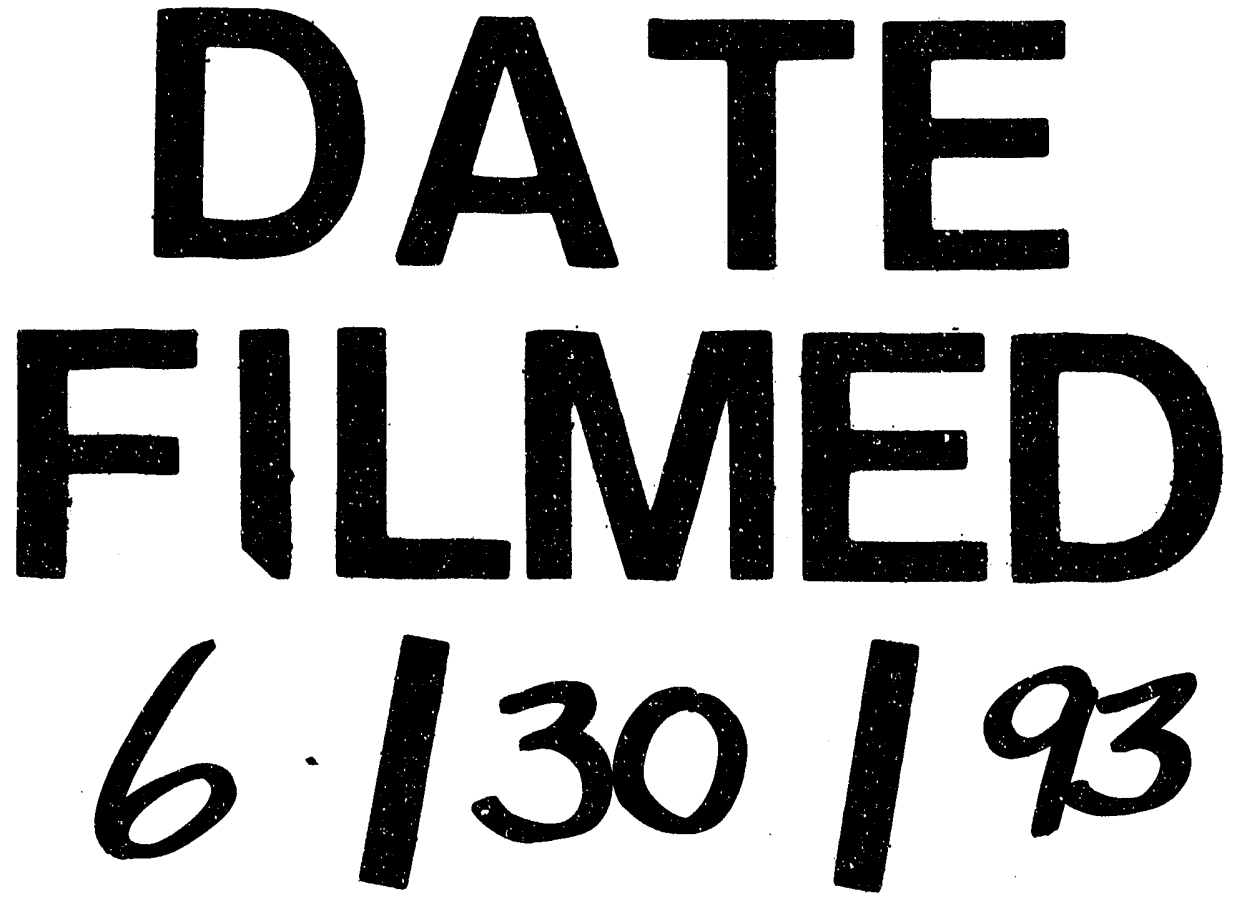
